ميلاد بور احمد'، جعفر اولادى 'و اصغر فلاح'

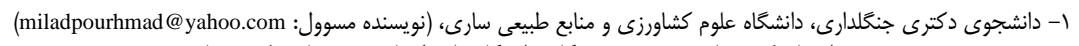

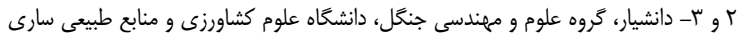

تاريخ دريافت: TV/T/MA

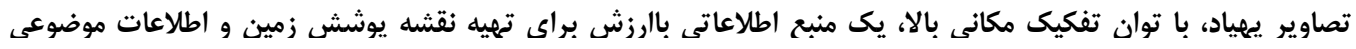

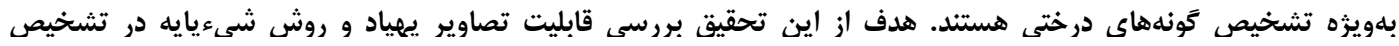

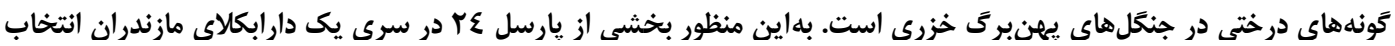

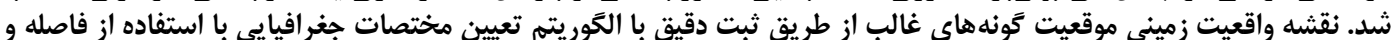

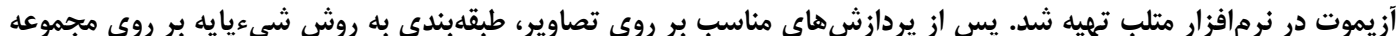

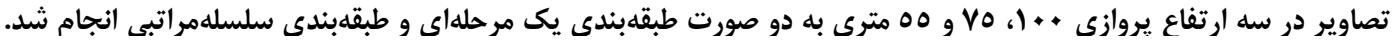

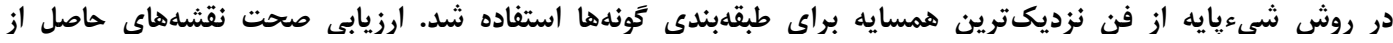

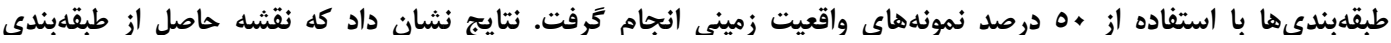

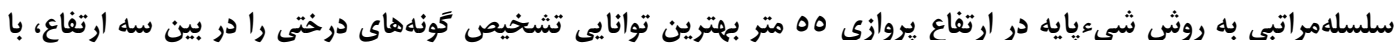

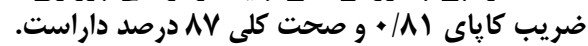

وازههاى كليدى: يهياد، تعيين كَونه، شىءيايه، طبقهبندى جنكَل

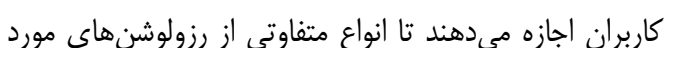

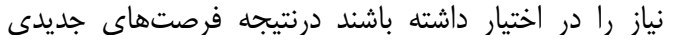

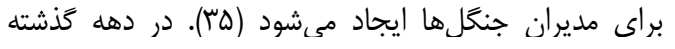

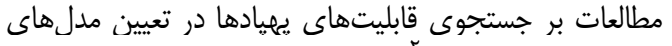

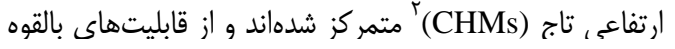

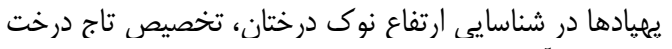

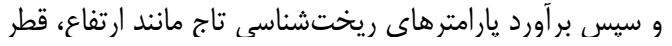

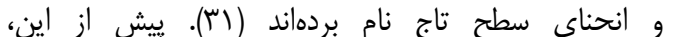

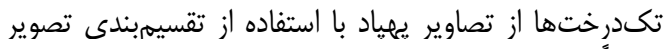

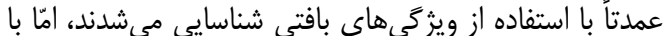

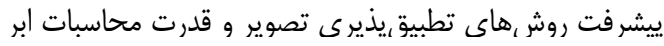

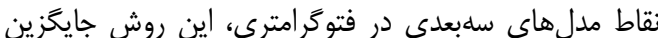

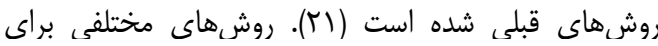

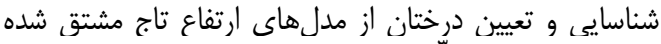

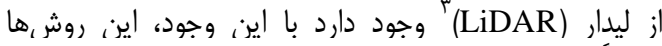

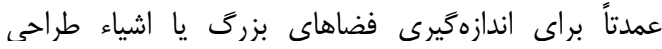

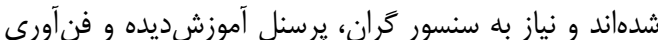

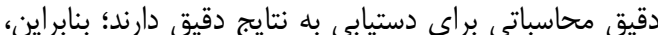

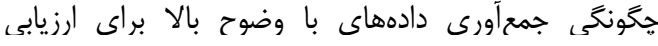

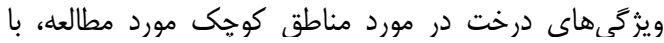

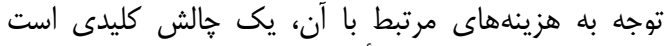

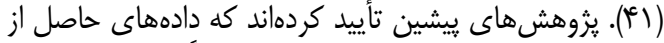

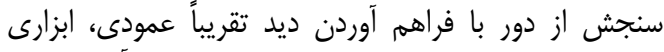

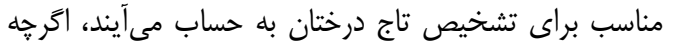

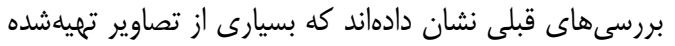

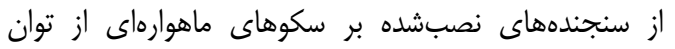

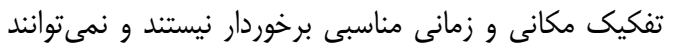

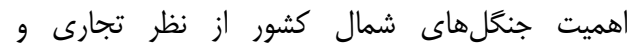
زيستمحيطى ايجاب مى كند كه همواره اطلاعات كمئ كمى و

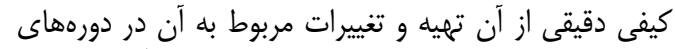

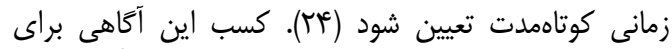

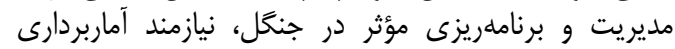

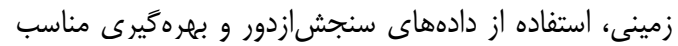

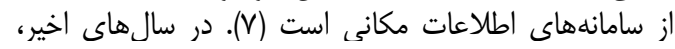

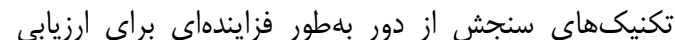

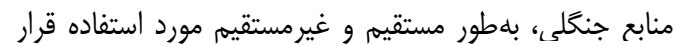

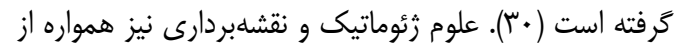

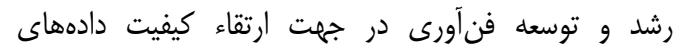

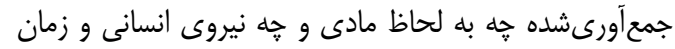

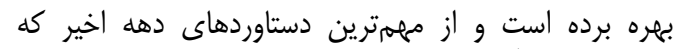

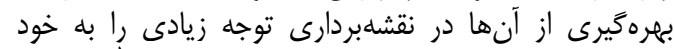

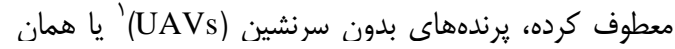

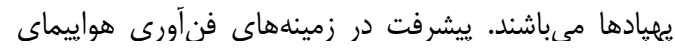

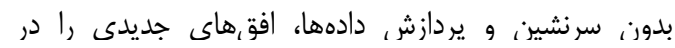

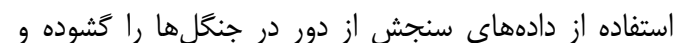

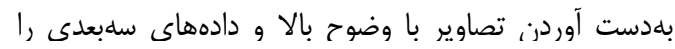

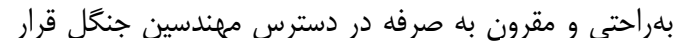

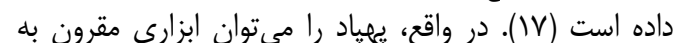

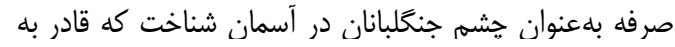

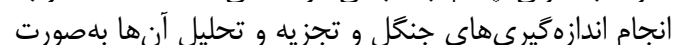

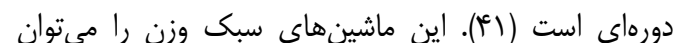

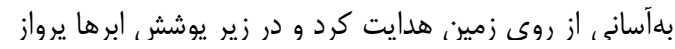

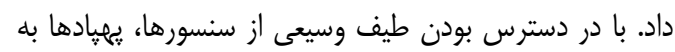




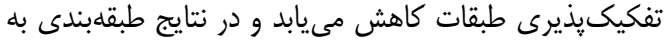

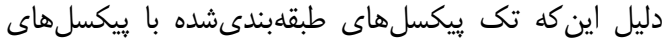

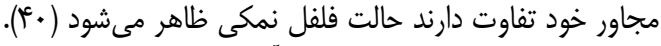

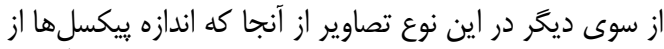

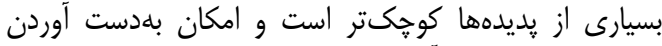

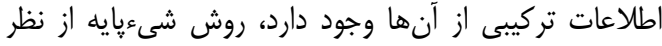

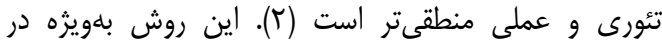

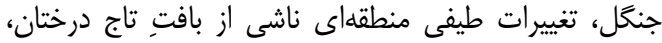

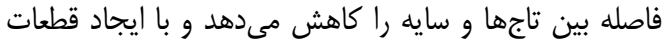

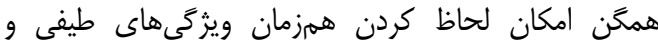

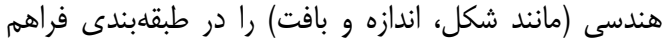

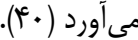

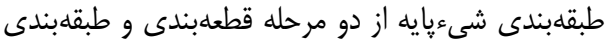

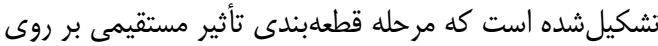

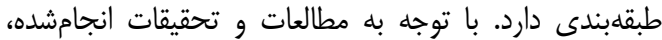

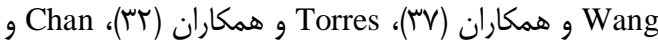

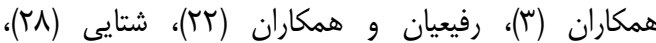

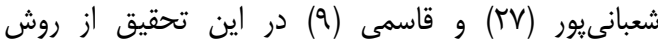

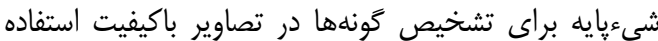

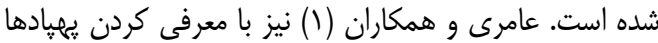

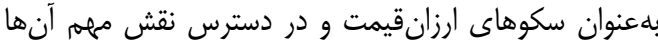

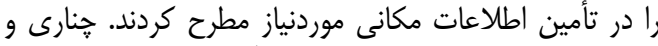

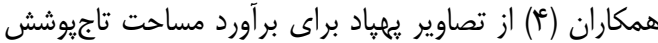

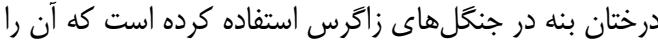

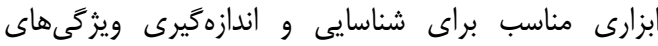

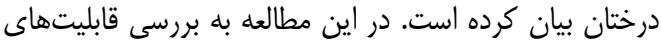

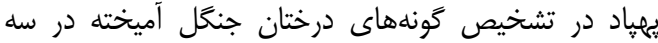

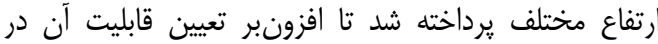

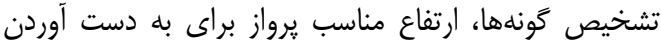

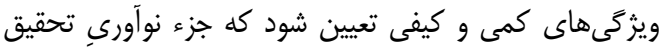

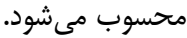

\section{مواد و روشها مهاد منطقه موردمطالعه}

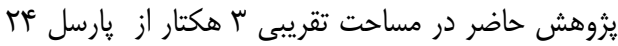

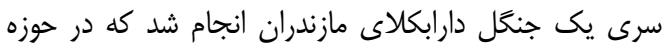

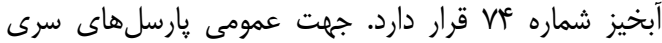

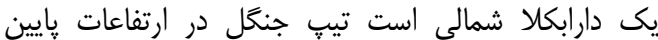

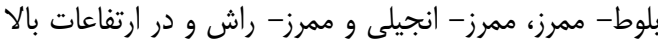
(بالاى • V متر) راش- ممرز يا راش خالص است.

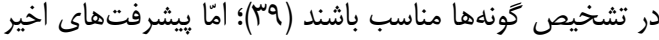

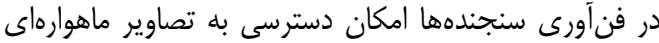

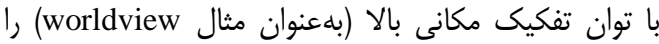

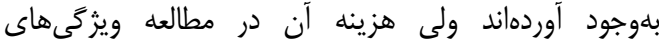

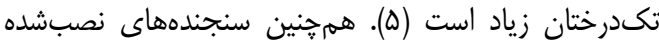

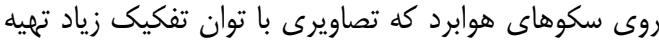

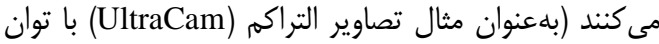

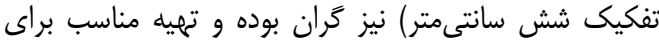

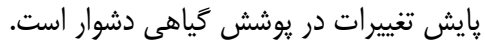

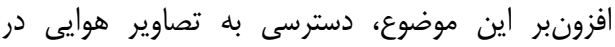

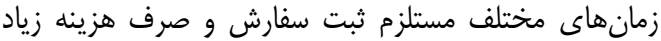

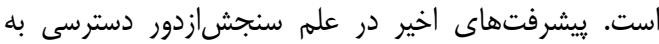

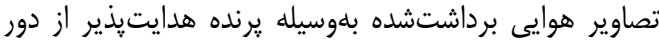

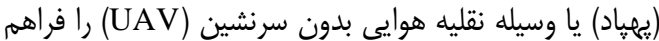

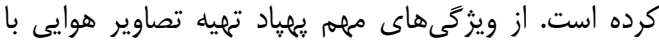

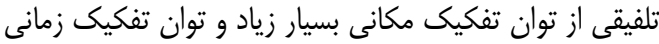

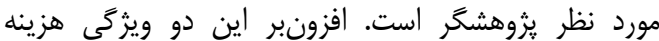
عملياتى و يِيجيدگى آن در مقايسه با ساير ابزارهاى اشارهشده

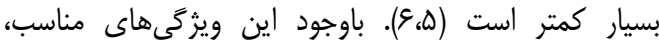

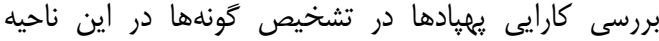

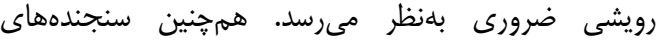

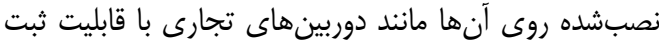

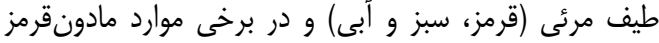

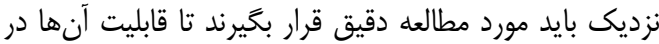

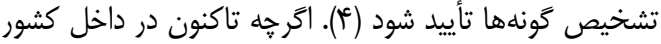

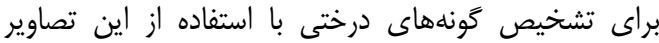

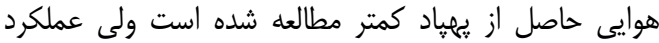

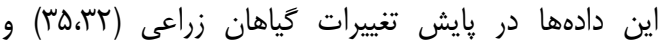

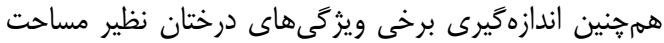

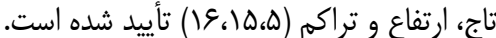

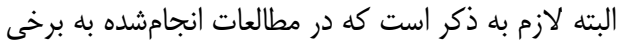

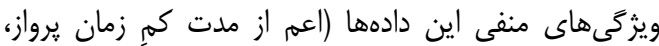

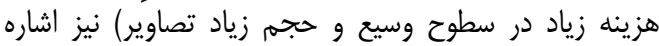

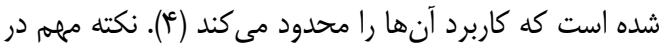

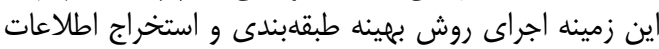

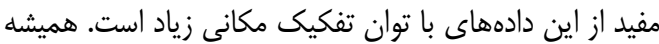

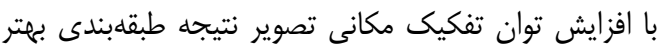

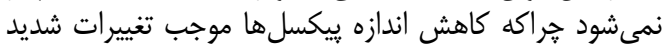

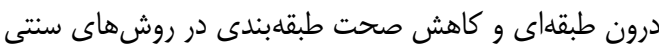

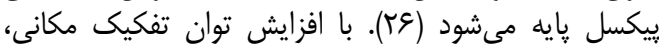

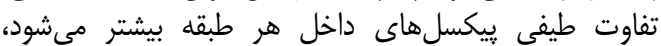




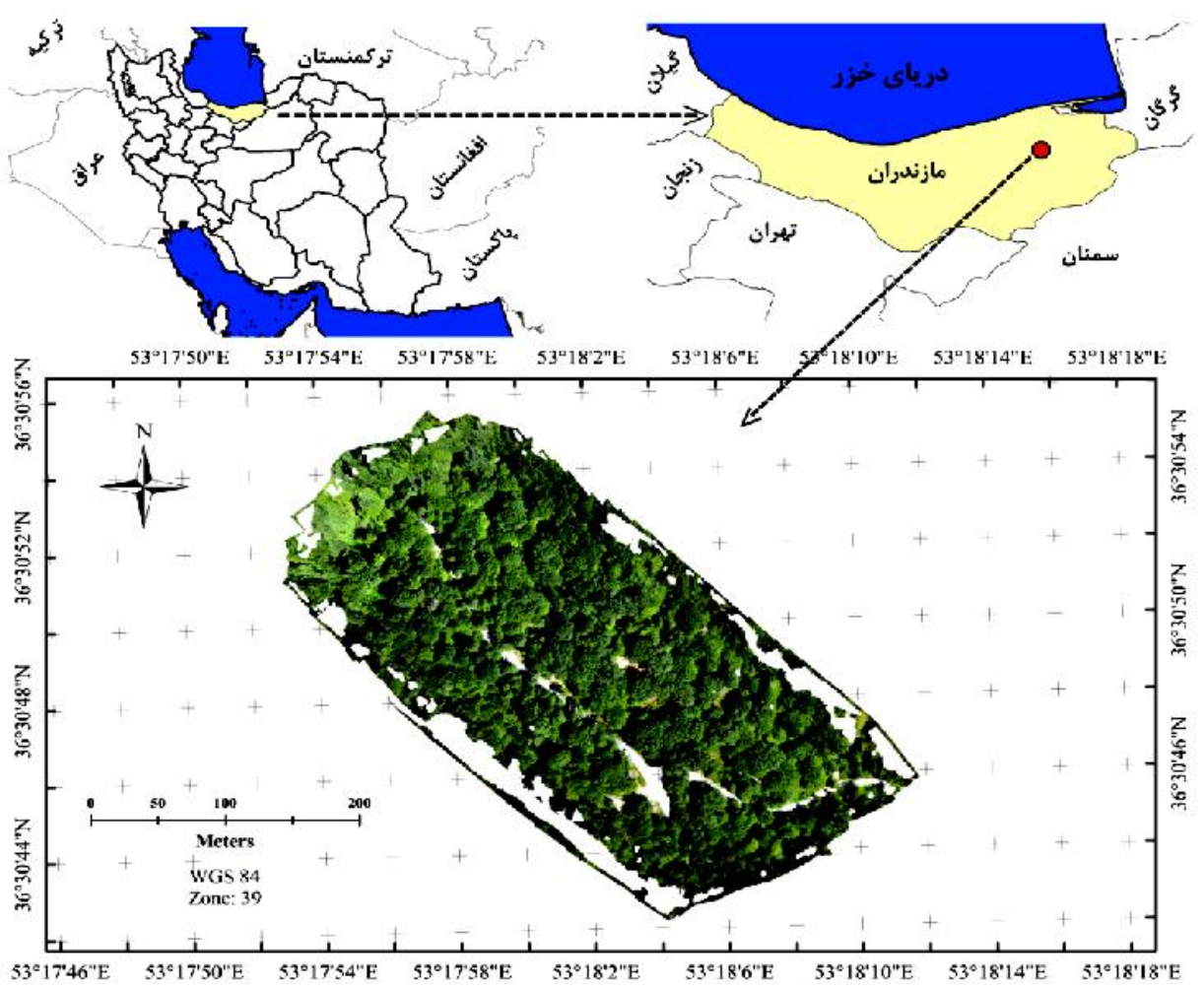

شكل 1- منطقه مورد مطالعه

Figure 1. Study area

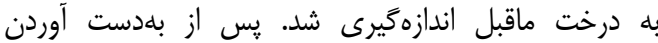

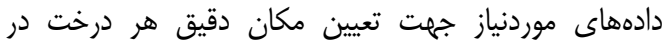

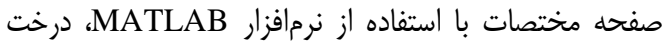

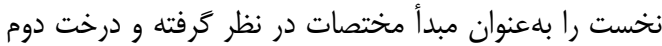

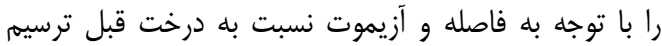

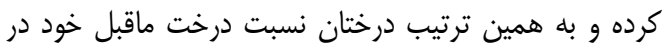

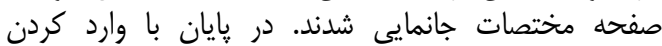
مختصات زمينى نقطه نخست، مختصات مختصات نقاط دران زمينى ساير

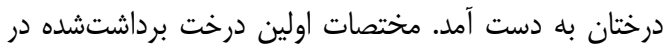

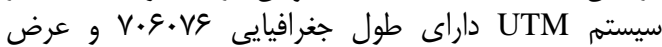

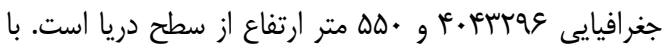

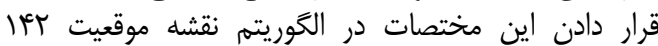

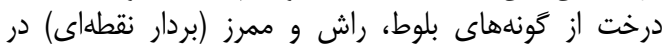

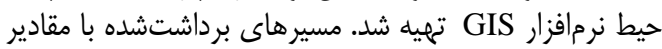

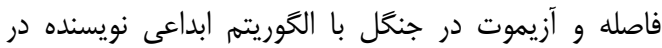

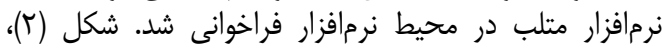

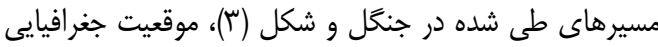
هريك از درختان را نشان مى دهد.
دادههاى مورداستفاده

نقشه واقعيت زمينى مورد

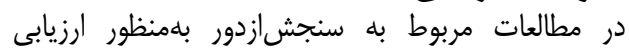

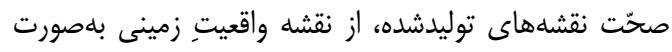

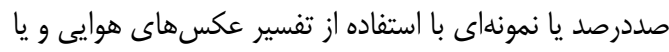

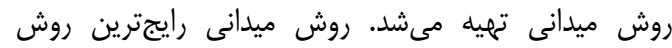

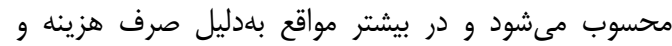

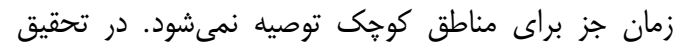

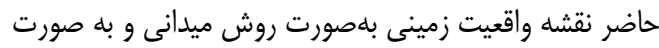

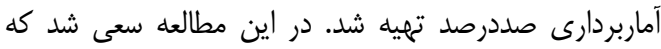

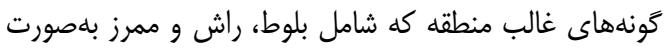

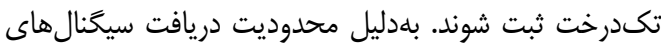

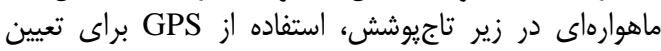

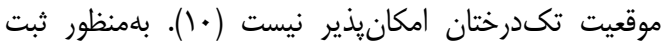

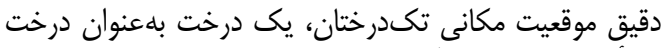

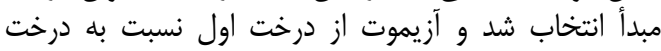

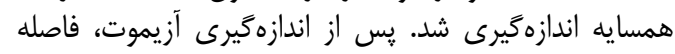

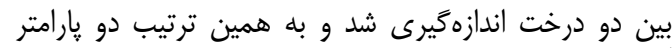
آزيموت و فاصله تمامى درختان در منطقه موردمطالعه نسبت ندان 


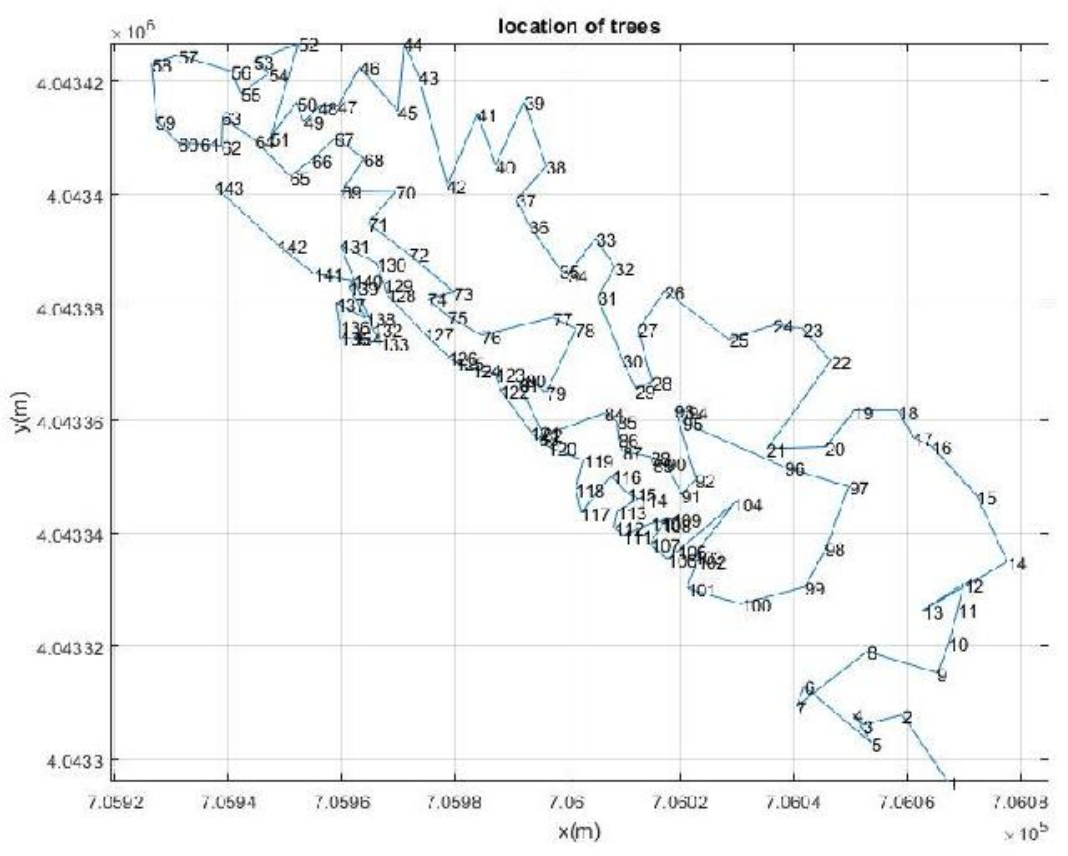

شكل r- مسيرهاى طى شده در جنكَل به منظور به دست آوردن موقعيت درختان

Figur 2. Tracks traversed in the forest to obtain location of trees

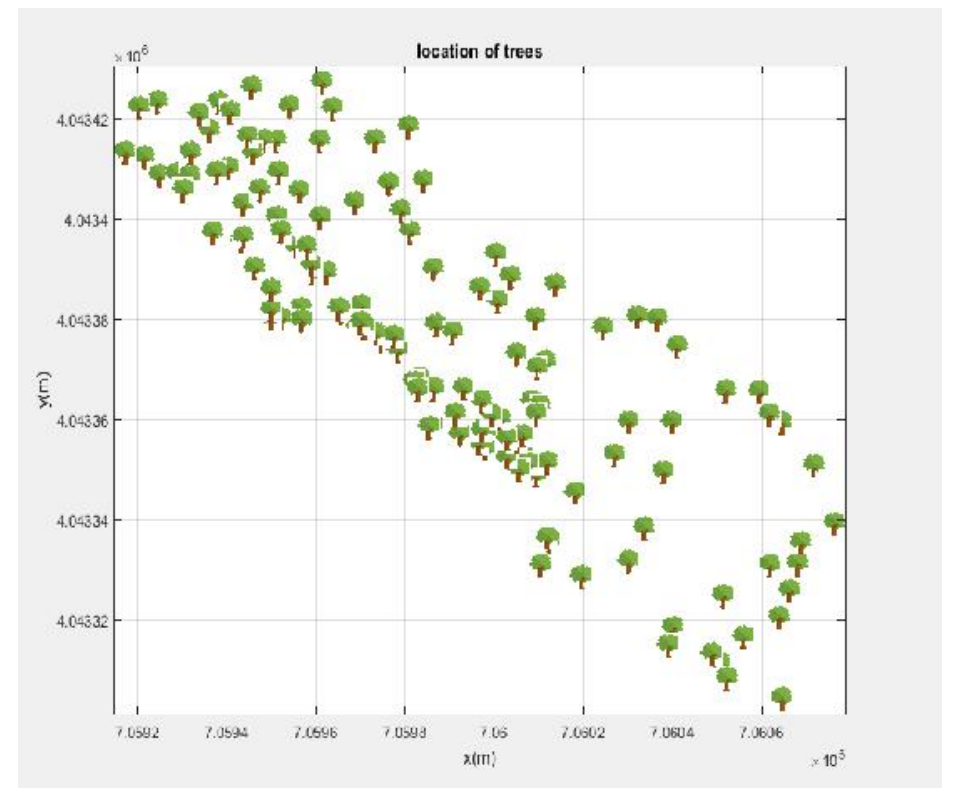

شكل س- موقعيت جغرافيايى درختان برداشتشده در منطقه

Figurt 3. Geographical location of trees in the area 


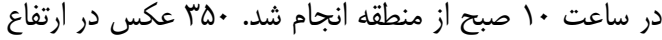

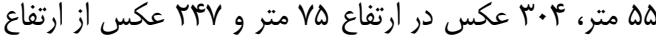

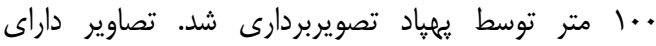

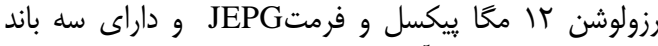

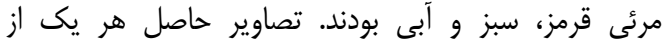

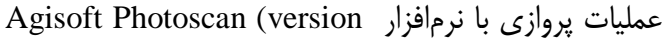
1.3.3 يردازش شد تا تصاوير حاصل در مراف مراحل بعدى مورد

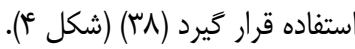

تصاوير يجياد

در اين مطالعه براى تهييه تصاوير از منطقه موردنظر از

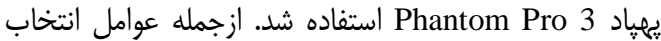

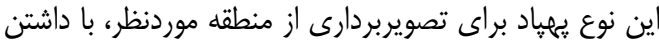

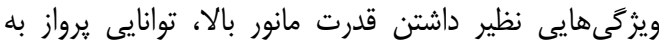

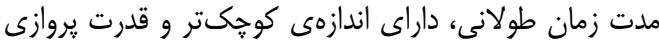

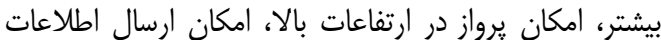

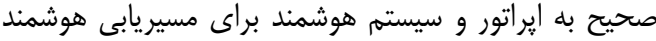

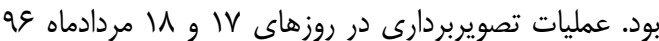

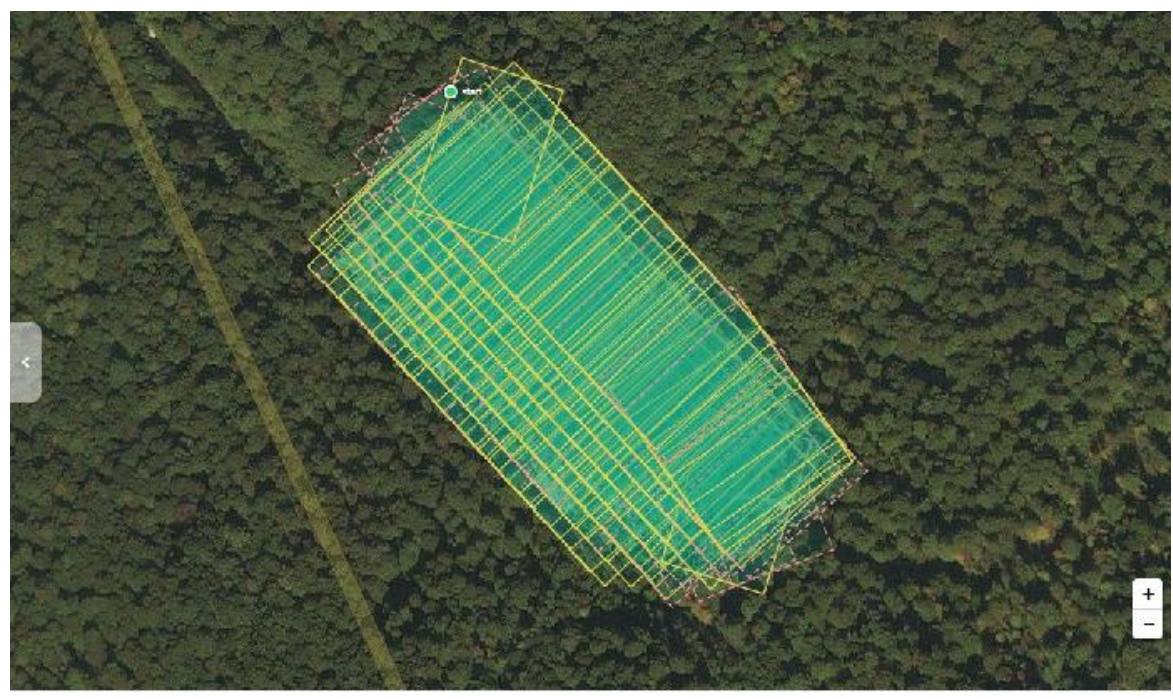

شكل عا- تصاوير برداشتشده از منطقه توسط يهياد

Figurt 4. Images taken from area by UAV

صحت جدا شد). نمونههاى تعليمى از گَونههاى درختى و از

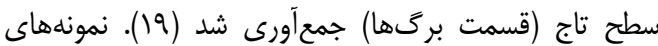

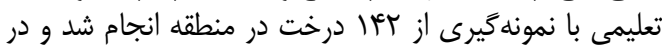
محيط GIS، نقشه حضور كونهانها در منطقه (واقعيت زمينى) بهدست آمد (شكل ها).
طبقهبندى

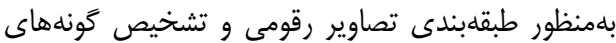

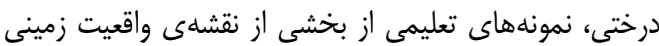

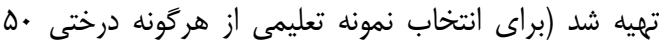

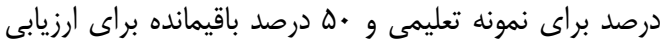




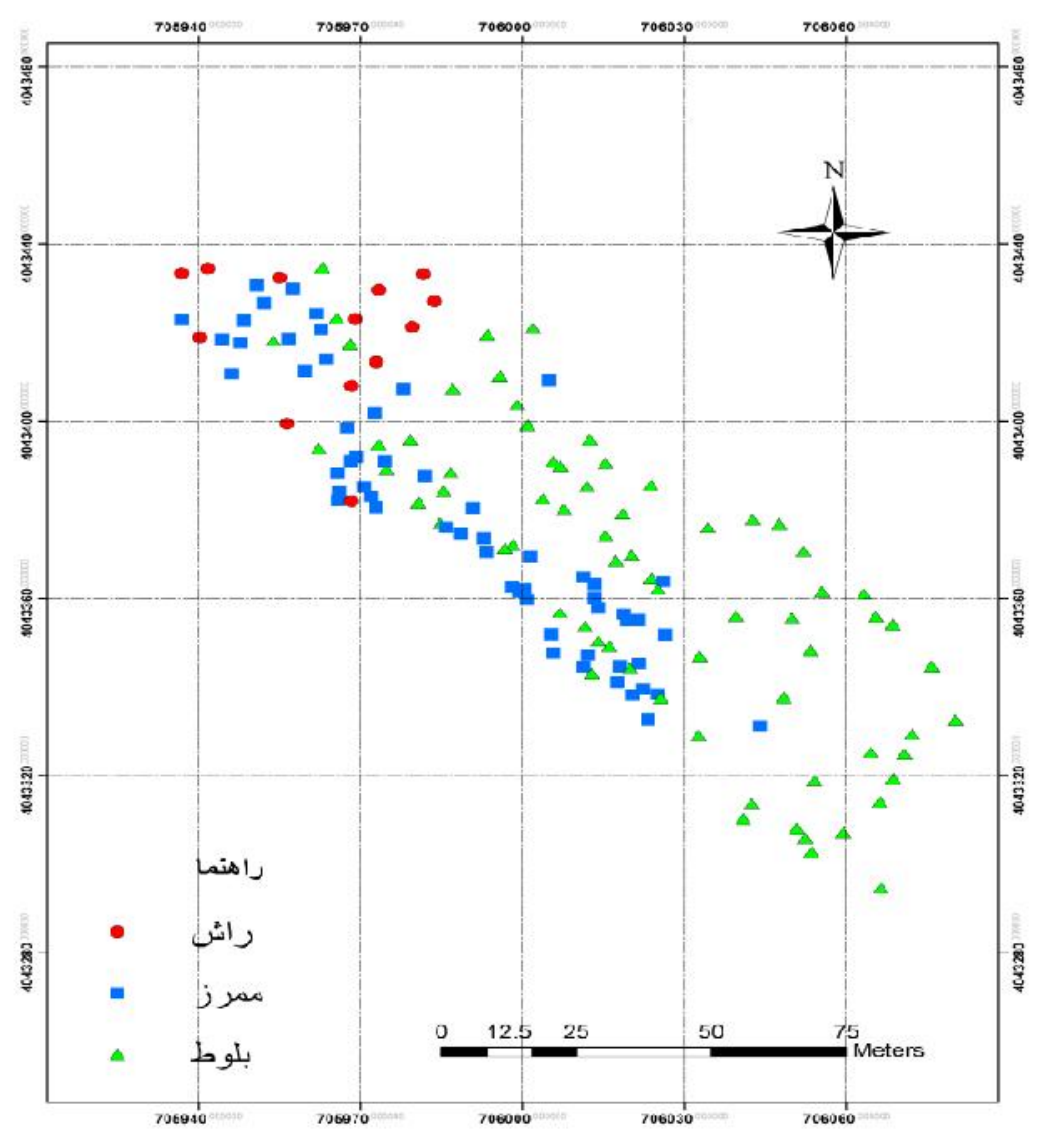

شكل ه- نقشه واقعيت زمينى

Figure 5. Ground truth map

شود. شاخصهاى قطعهبندى (رنخى، شكل، فشردگىى، هموارى

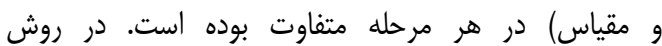

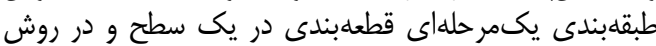

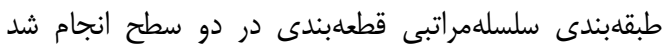

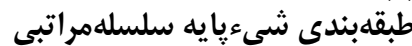

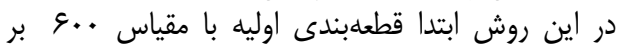

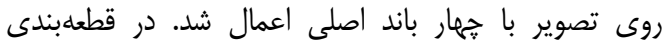

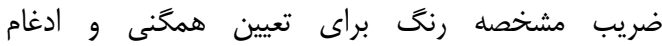

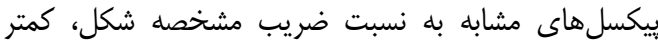

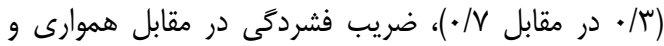

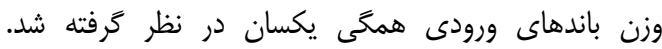

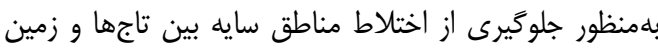

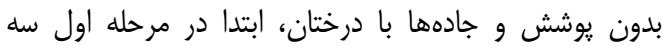

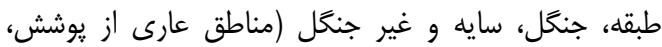

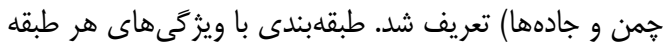

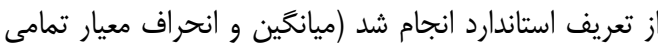

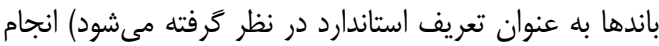

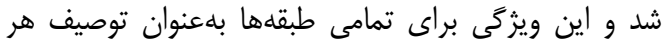

طبقهبندى شى شِيايه (Object-Based)

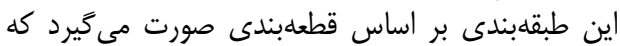

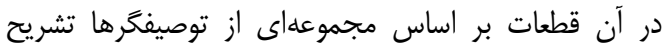

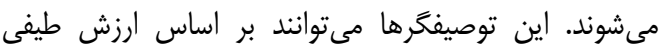

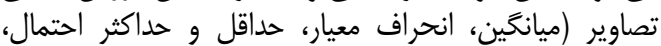

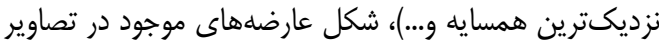

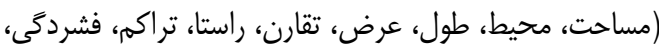

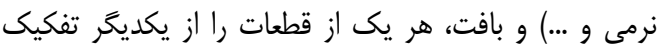

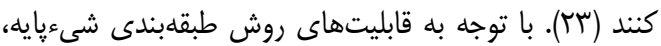

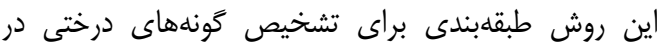

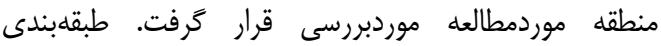

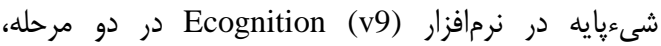
قطعهبندى و طبقاينه در نرمافزي انجام شد.

قطعهبندى (Segmentation)

در اين تحقيق براى هر دو طبقلبندى (سلسلهمراتبى و

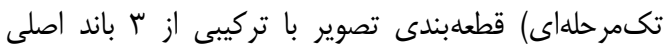

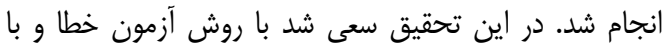

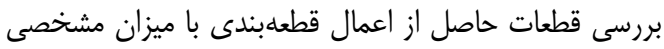

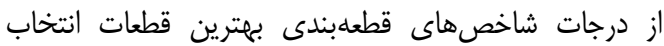




\section{طبقلبندى شىء پايه يكمر حلهاى}

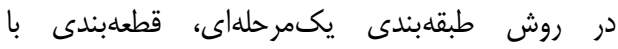

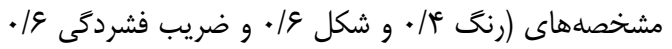

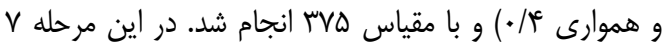

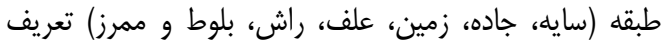

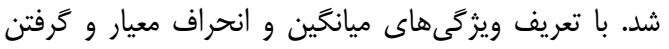

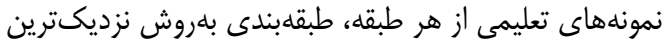

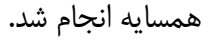

曰-

- classes

Forest

Beech

Hornbeam

Oak

Nonforest

Grass

Ground

Road

Shadow

ب) كلاسهها در طبقهبندى سلسلهمراتبى

Figure 6. A) Classes in One-Step classification

نتايج و بحث

نتايج طبقهبندى شىعيايه از تصاوير بهيهاد شامل مراحل

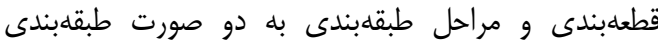

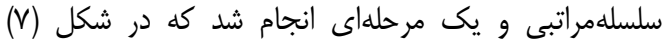

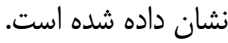

طبقه استفاده و طبقهبندى با طبقات سايه، جنگل و غير جنكَل

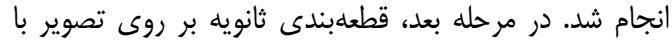

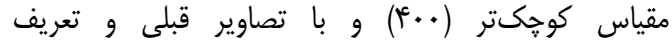

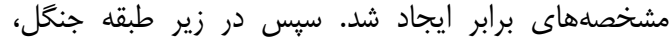

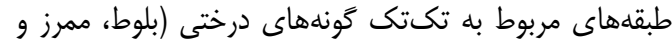

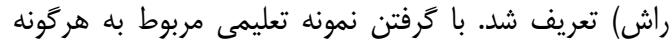

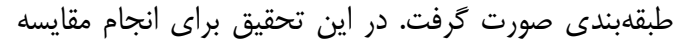

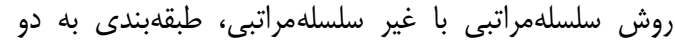

صورت انجام شد (1) (1).

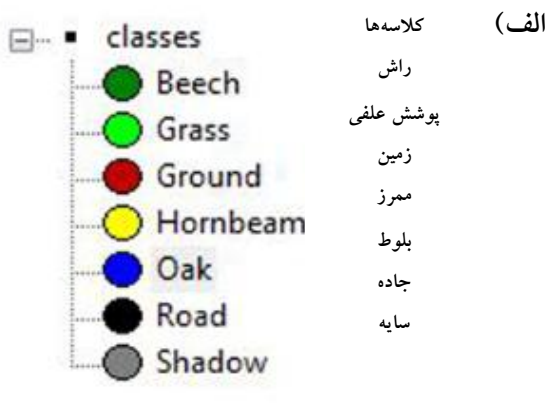

شكل و- الف) كلاسهها در طبقهبندى يك مرحلهاى

B) Classes in Hierarchical classification

\section{صحت نتايج طبقلهبندى}

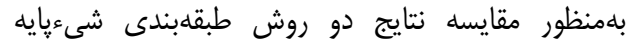

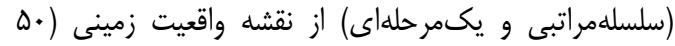

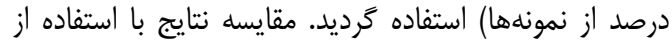
معيار ضريب كايا و صحت كلى انجام شد. 


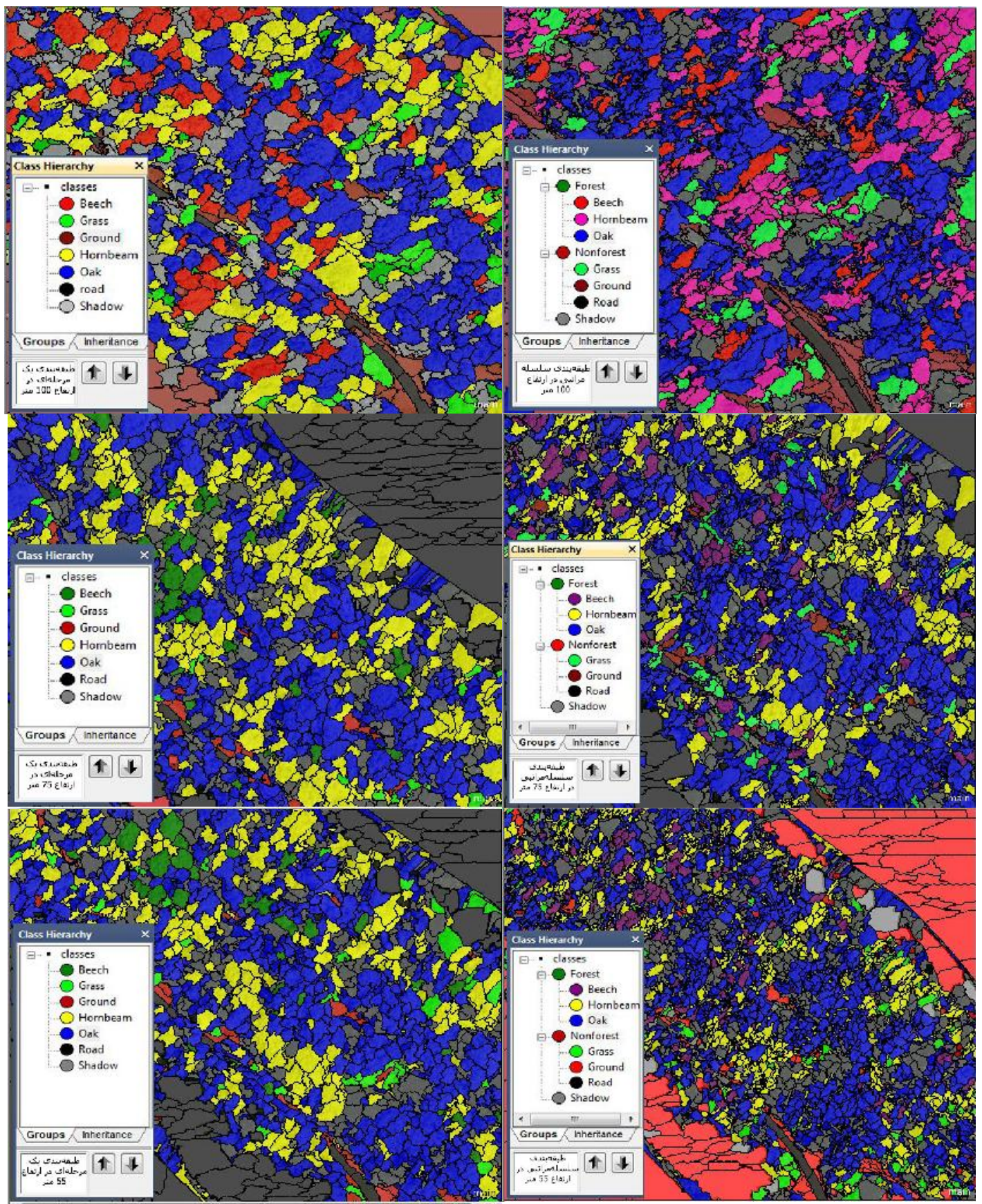

شكل V- طبقابندى يكمرحلهاى و سلسلهمراتبى در سه ارتفاع يروازى

Figure 7. One-step and Hierarchical classification in three flight height 


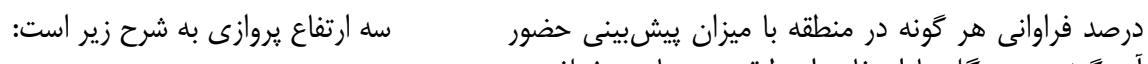

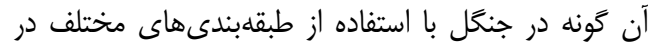

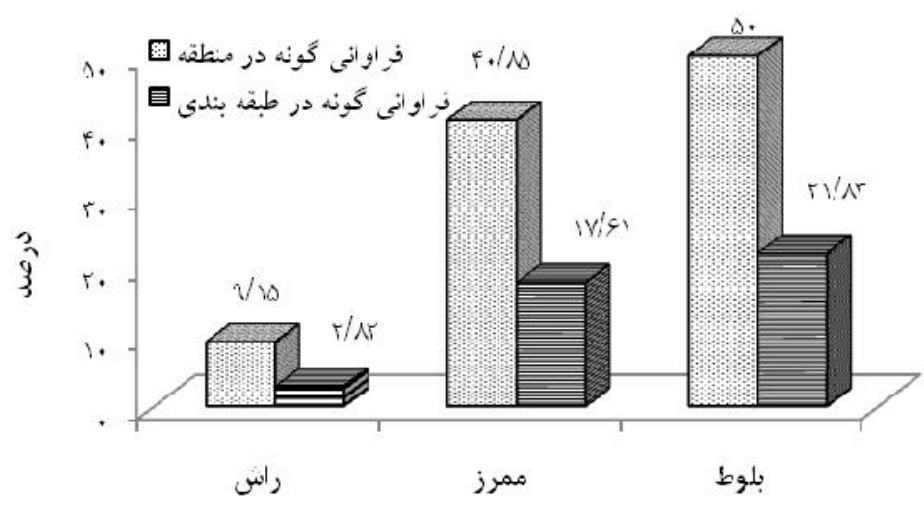

شكل ^- هيستوكر ام ارزيابى صحت طبقهبندى كونهها در طبقابندى يكمر حلهاى در ارتفاع برواز ..1 متر

Figure 8. Accuracy histogram of species classification in One-Step classification at $100 \mathrm{~m}$ flight height

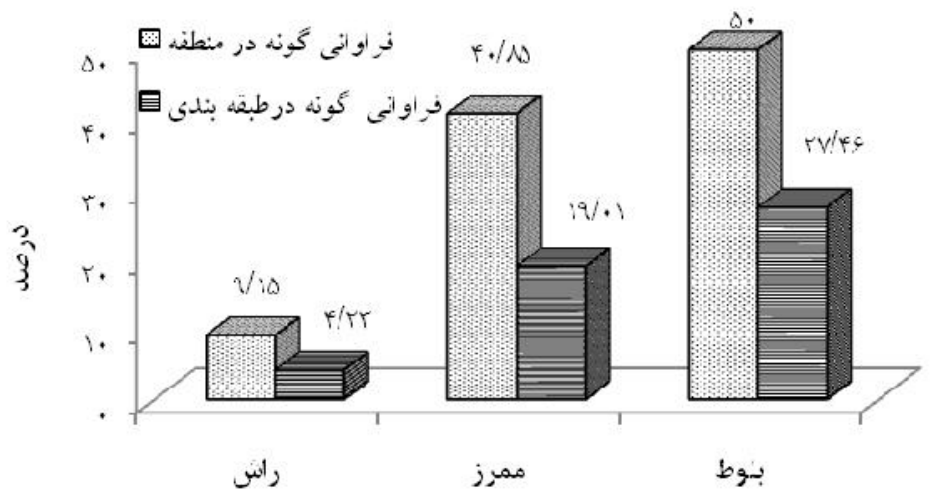

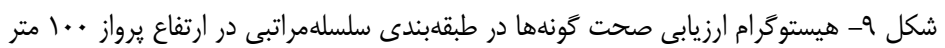

Figure 9. Accuracy histogram of species classification in Hierarchical classification at $100 \mathrm{~m}$ flight height

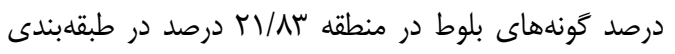
يكمرحلهاى و و \&

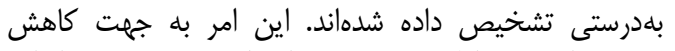

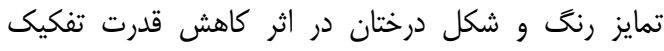
تصوير است كه با افزايش ارتفاع تصويربردارى رن دان داده است.

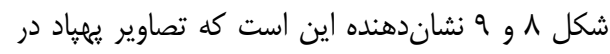

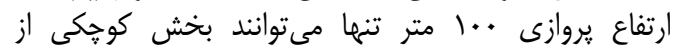

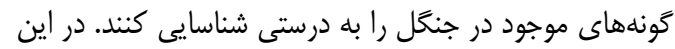

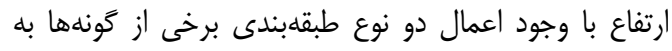

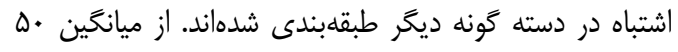


$\checkmark \cdot$ شناسايى گَونهاى درختى در تودهاى يهنبرى آميخته جنكَل هاى خزرى با استفاده از تصاوير يهياد

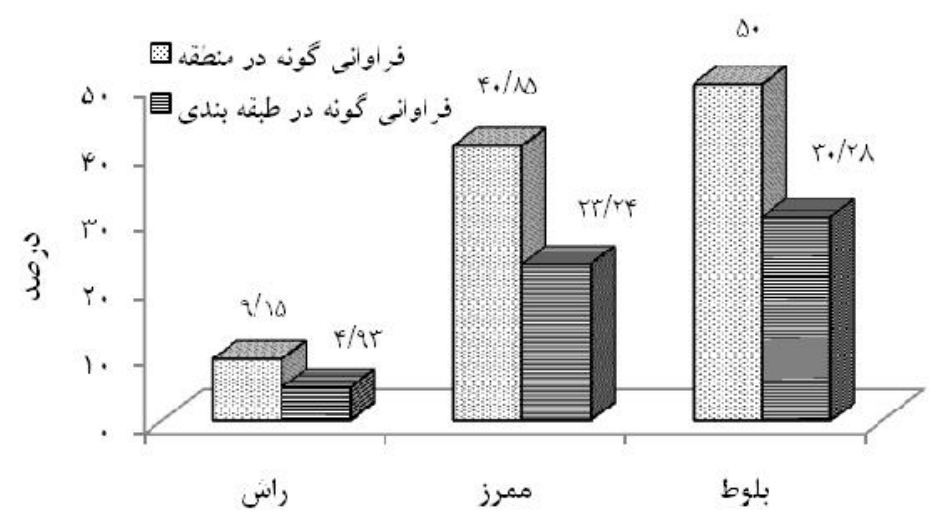

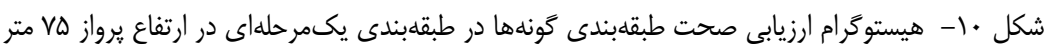

Figure 10. Accuracy histogram of species classification in One-Step classification at $75 \mathrm{~m}$ flight height

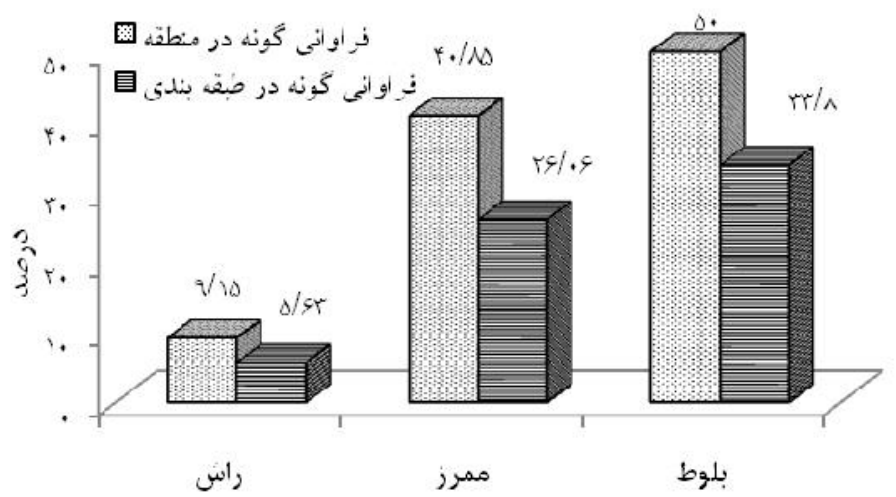

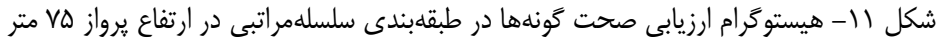

Figure 11. Accuracy histogram of species classification in Hierarchical classification at $75 \mathrm{~m}$ flight height

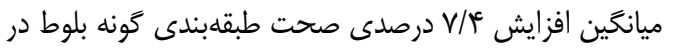

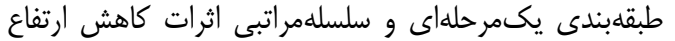

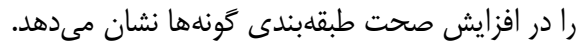

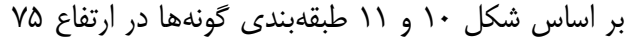

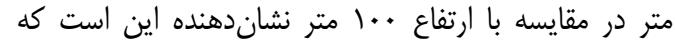

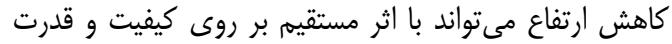
تفكيك تصاوير بر تشخيص گونهاعها مؤثر باشد.

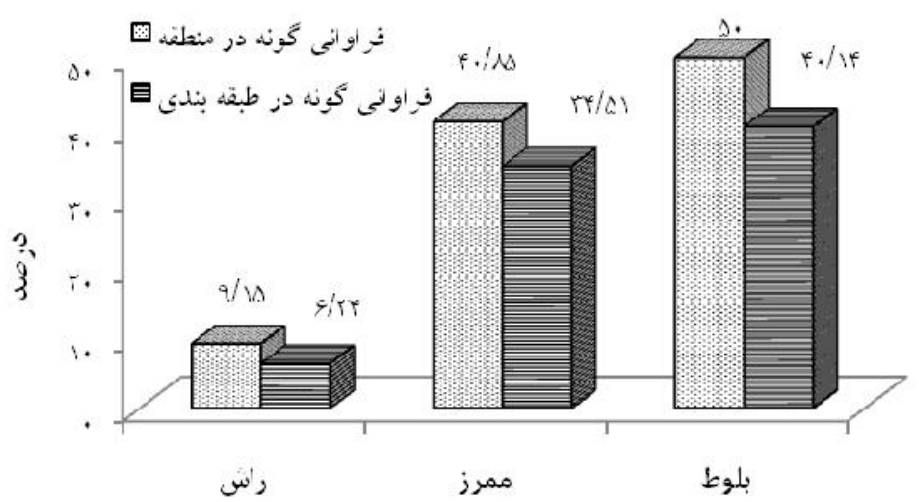

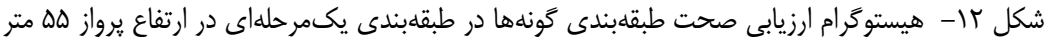

Figure 12. Accuracy histogram of species classification in One-Step classification at $55 \mathrm{~m}$ flight height 


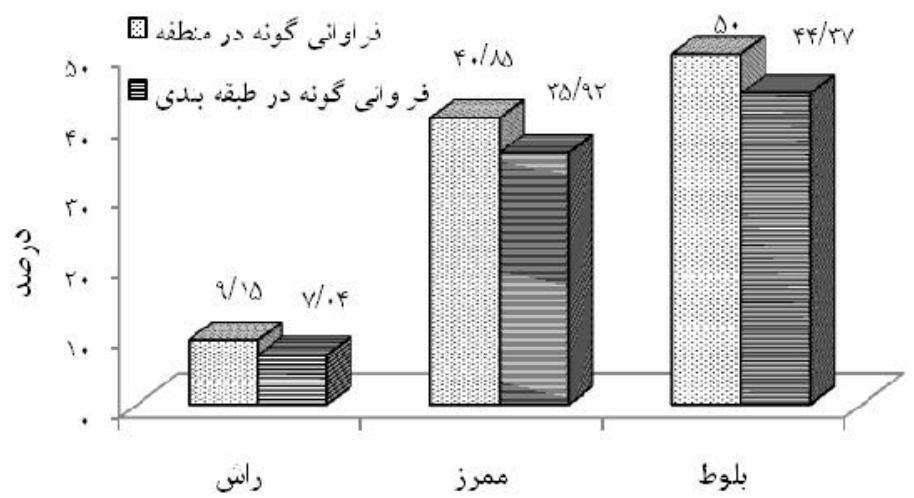

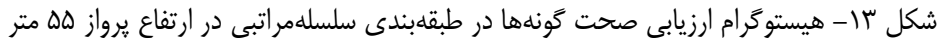

Figure 13. Accuracy histogram of species classification in Hierarchical classification at $55 \mathrm{~m}$ flight height

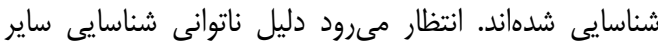

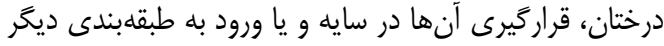

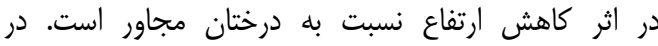

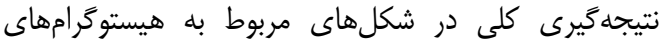

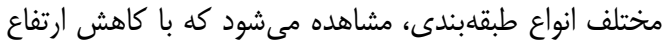

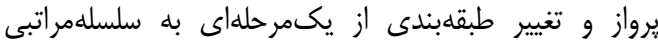

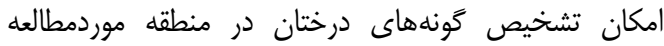

بيشتر مىشود.
در شكل r | و سا طبقلبندى در ارتفاع ها متر مشاهده

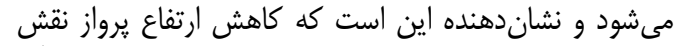

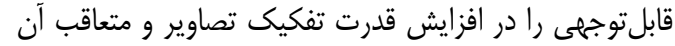

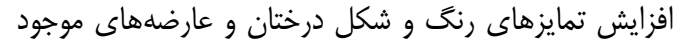

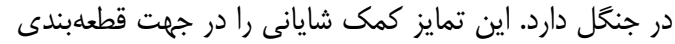

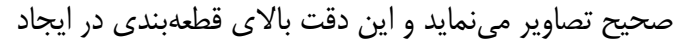

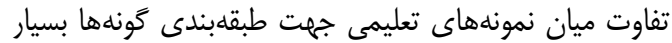

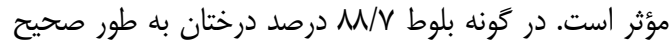

Table 1. Kappa coefficients for One-step classification at different flight heights

\begin{tabular}{|c|c|c|c|c|}
\hline صحت كلى & سطح معنى دارى & خطاى استاندارد & ضريب كايا & ارتفاع در طبقهبندى دومرحلهاى \\
\hline FT/TD & $\%$ &.$/ . \mathrm{HT}$ & . MGT & $1 \ldots$ \\
\hline$\Delta \Lambda / \& \Delta$ & $\cdot / \ldots$ & 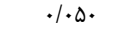 & . 1490 & VD \\
\hline $1 . / 99$ &.$/ \ldots$ & $.1 . \Delta F$ & g & $\Delta \Delta$ \\
\hline
\end{tabular}

جدول r- ضرايب كايا براى طبقهبندى سلسلهمراتبى در ارتفاعات مختلف Table 2. Kappa coefficients for Hierarchical classification at different flight heights

\begin{tabular}{|c|c|c|c|c|}
\hline صحت كلى & سطح معنىدارى & خطاى استاندارد & ضريب كايا & ارتفاع در طبقهبندى دومرحلهاى \\
\hline$\Delta \cdot / V \cdot$ & $\cdot / \cdots$ & .1 .94 & . $/ F T D$ & $1 \cdots$ \\
\hline 9Q/AT & $\%$ & $\cdot 1 \cdot \Delta \Delta$ &.$/ 9 \vee G$ & VQ \\
\hline AV/RT &.$\ldots$ & $.1 . \uparrow \wedge$ & ./N| & $\Delta \Delta$ \\
\hline
\end{tabular}

مىتواند اين امكان را فراهم سازد تا تحقيقات از سطح توده

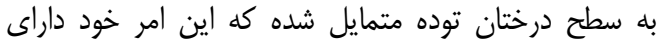

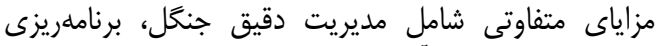

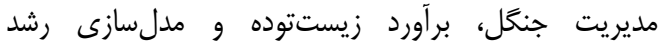

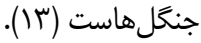

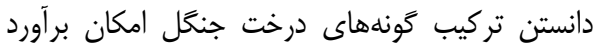

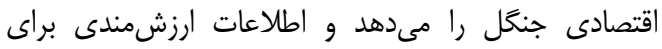

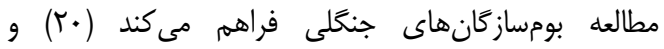

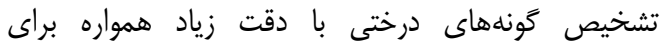

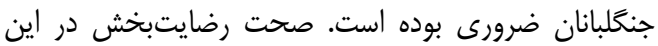

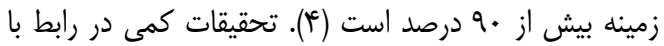

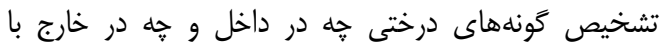

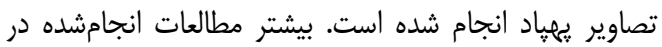

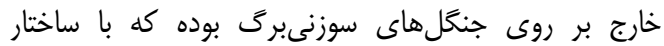

ضريب كايا و تحليل آمارى مبتنى بر آن اندازهاى

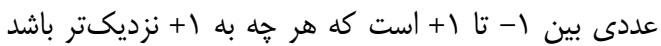

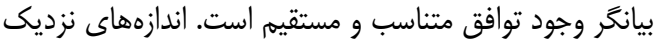

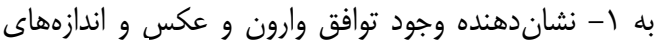

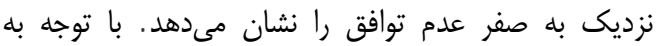

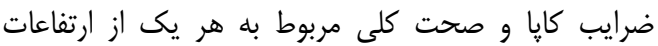

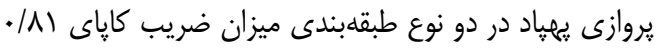

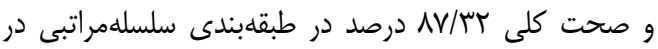

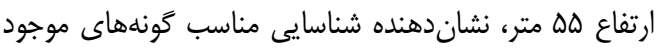

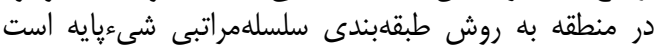

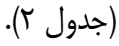
افزايش سطح جزئيات جنگل مئلوانداند مدلسازى دقيق

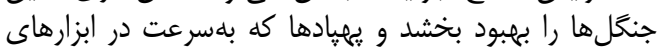

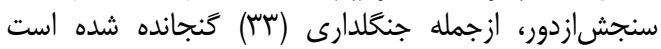




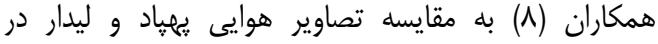

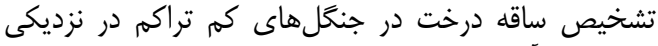

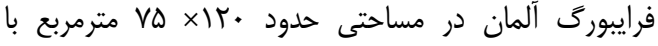

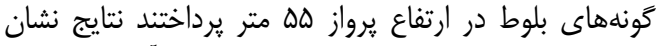

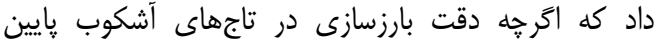

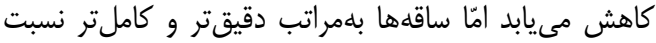

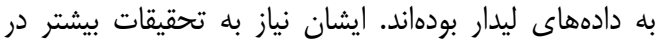

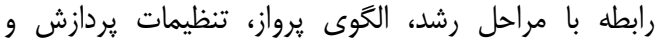
استفاده از اطلاعات طيفى راضرورى دانئ دانستند.

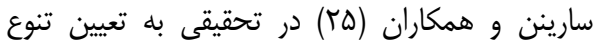

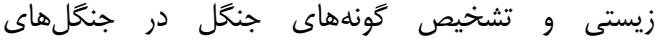

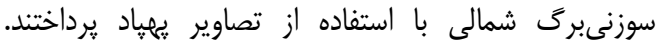

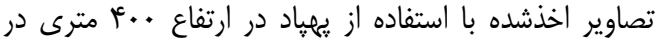

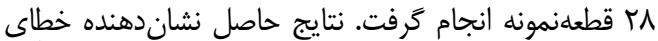

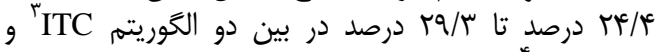

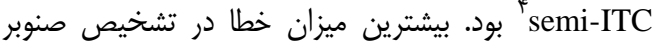

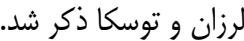

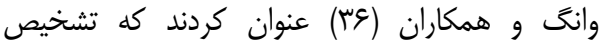

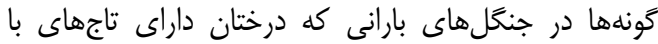

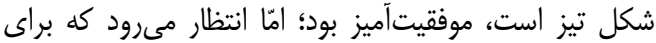

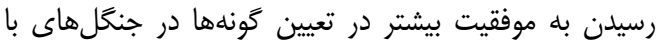

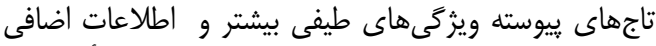

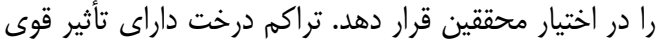

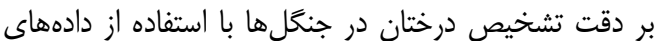

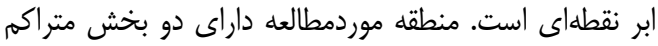

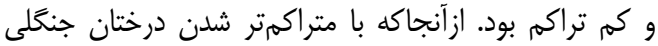

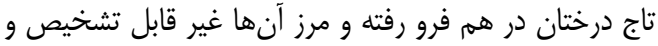

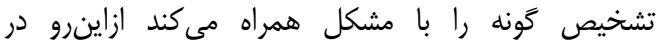
بخشهاى متراكم تشخيص كَونه مشكل و با خطاى بيشترى

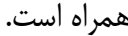

اين امر در صورت وجود آشكوبهاى متفاوت و ماتو

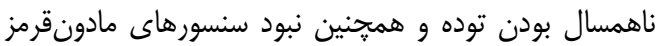

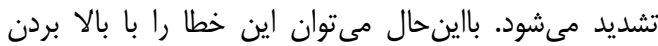

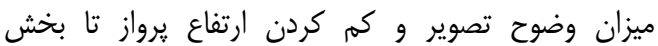

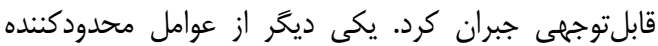

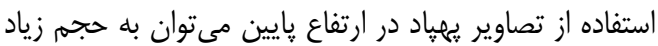

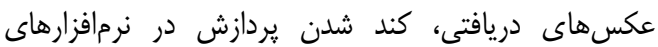

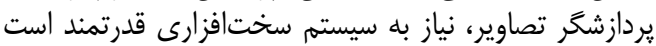

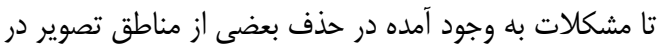

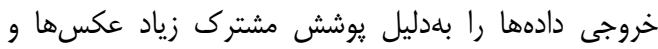

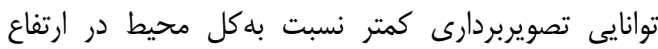

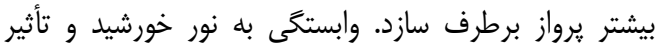

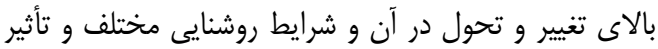

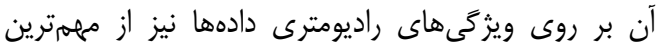

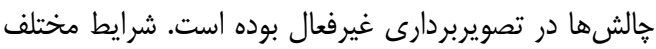

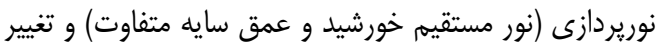
نور در تاجيوشش درختان كه ميتوتواند باعث سائ سايه و روشن

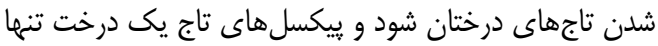

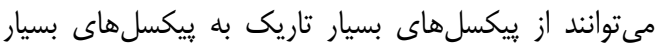

1- Object-Based Change Detection

3- Individual tree crown approach
جنگَلهاى ايران متفاوت است. هدف از اين تحقيق، بررسى

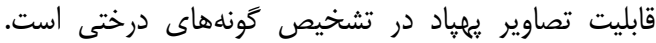

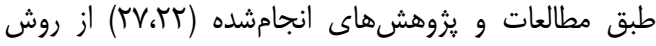

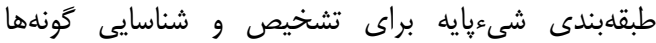

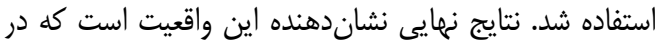

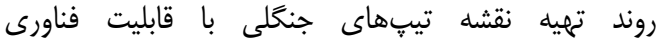

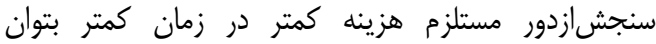

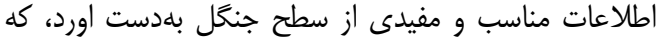

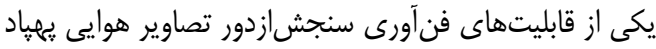

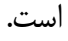
در اين مطالعه براى مقايسه و ارزيابى از روشهاى

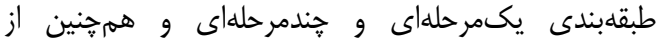

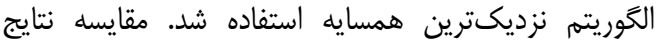

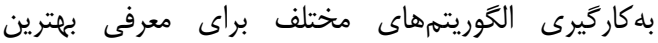

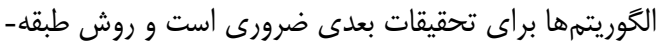

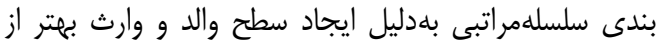

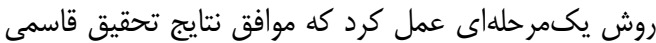

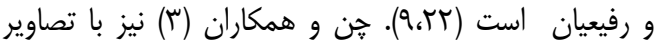

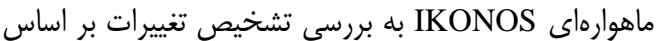

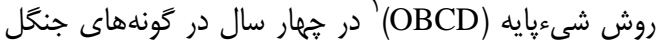

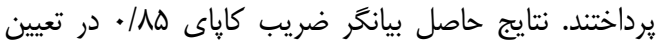

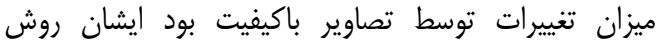

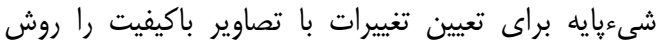

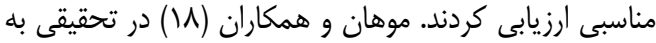

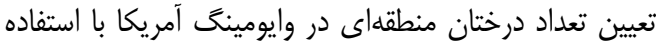

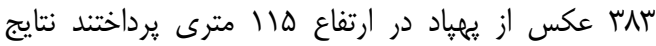

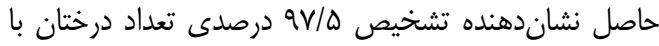

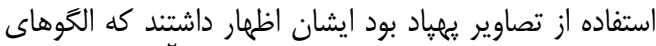

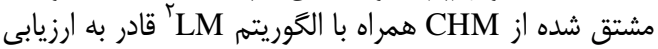

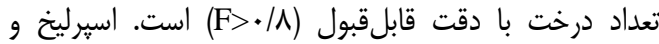

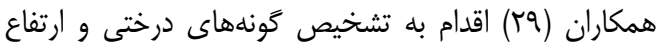

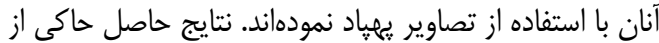

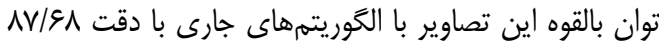

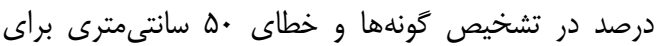
برآورد ارتفاع درختان بود تا نشان از عملكرد إمدان اميدواركننده اين براي

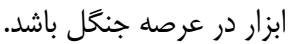

با توجه به نتايج تحقيق اسيرليخ و همكاراران (وبارئ،

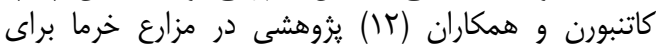

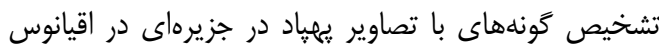

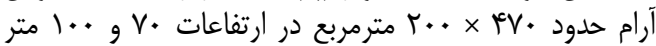

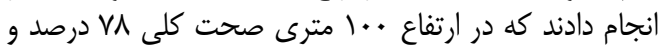

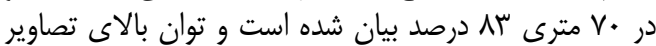

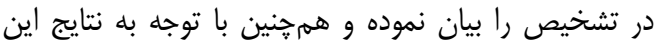

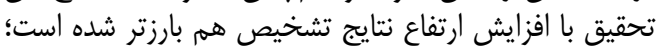
البته بايد مطالعات بيشترى در رابطه با افزايش إنائ ارتفاع و شرايط

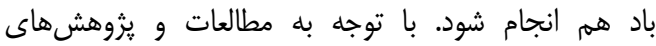

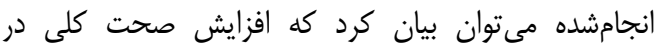

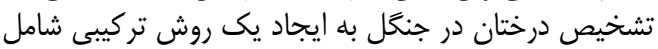

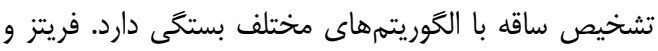




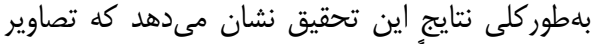

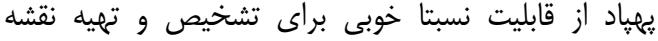

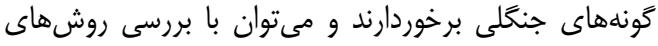

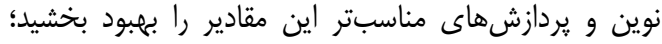

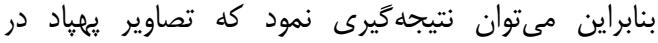

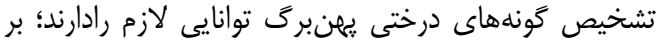

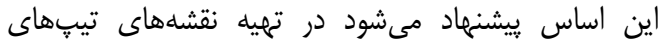

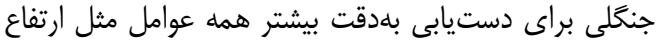

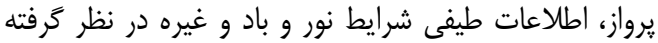

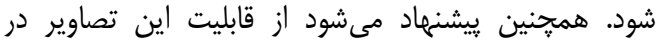

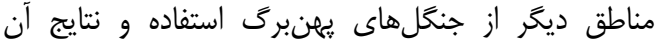

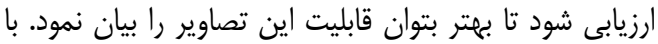

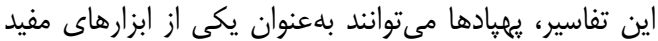

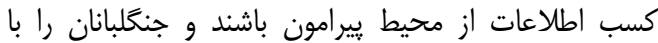

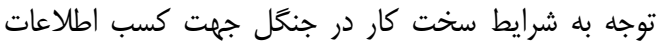
لازم، يارى كنند.
روشن تغيير يابد و بايد در تجزيلوتحليل دادهها در يك محيط

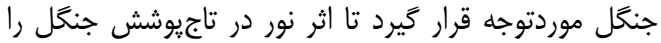
كاهش دهد (1) - (1).

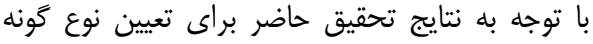

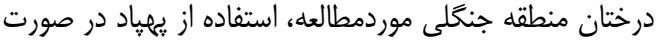

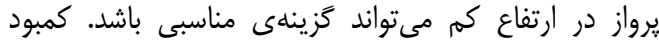

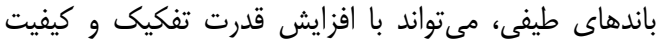

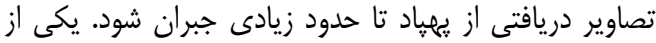

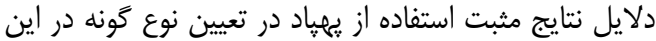

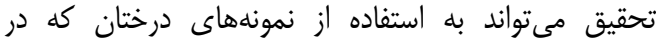

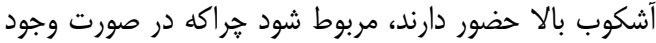

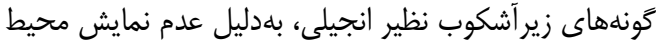

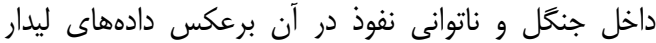

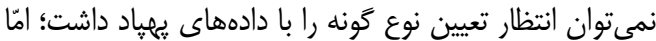

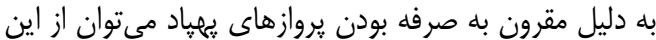

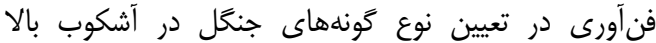

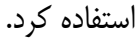

منابع

1. Ameri, A., F. Dadras Javan and N. Zareinpanche. 2017. A review of methods for extracting roads from UAV aerial images. Geospatial Engineering Journal, Iranian Society for Surveying and Geomatic Engineering, 4(8): 57-65 (In Persian).

2. Benz, U.C., P. Hofmann, G. Willhauck, I. Lingenfelder and M. Heynen. 2004. Multi-resolution, Object-oriented fuzzy analysis of remote sensing data for GIS-ready information. ISPRS Journal of photogrammetrv and Remote Sensing. 58(3-4): 239-258.

3. Chen. G.. G.J. Hav. L.M. Carvalho and M.S. Wulder. 2012. Object-based change detection. International Journal of Remote Sensing, 33(14): 4434-4457.

4. Chenari, A., S.Y. Erfanifard, M. Dehghani and H. Pourghasemi. 2017. Estimation of crown area of wild pistachio single trees using DSM of UAV aerial images in Baneh Research Forest, Fars province. Journal of Wood and Forest Science and Technology, 24(4): 117-130 (In Persian).

5. Chianucci, F., L. Disperati, D. Guzzi, D. Bianchini, V. Nardino, C. Lastri, A. Rindinella and P. Corona. 2016. Estimation of canopy attributes in beech forests using true colour digital images from a small fixed-wing UAV. International J. Applied Earth Obsevation and Geoinformation, 47: 60-68.

6. Colomina, I. and P. Molina. 2014. Unmanned aerial systems for photogrammetry and remote sensing. ISPRS J. of Photogrammetry and Remote Sensing, 92: 79-97.

7. Erfanifard, Y., J. Feghhy, M. Zobeiry and M. Namiranian. 2010. Review Possible Provision Maps Canopy Density In The Forest Using Aerial Imaging And GIS, Proceeding of The National of Eighth National Conference Geomatics (In Persian).

8. Fritz. A.. T. Kattenborn and B. Koch. 2013. UAV-based photogrammetric point clouds-Tree stem mapping in open stands in comparison to terrestrial laser scanner point clouds. Int. Arch. Photogramm. Remote Sensing Information Science, 40: 141-146.

9. Ghasemi, A., Sh. Shataee and J. Mohamadi. 2017. Identify Tree Species In Mixed Hardwood Stand of The Caspian Forests Using Digital Images Aerial Cameras Ultracam-D. Journal of Wood and Forest Science and Technologv. 24(1): 77-90 (In Persian).

10. Hutchens. C.L.. B.R. Sarbin. A.C. Bowers. I.D. McKillican. K.K. Forrester and R.M. Buehrer. 2008 An improved method for GPS-based network position location in forests. In Wireless Communications and Networking Conference, 2008. WCNC 2008. IEEE (pp: 273-277) IEEE.

11. Kane, V.R., A.R. Gillespie, R. McGaughey, J.A. Lutz, K. Ceder and J.F. Franklin. 2008 Interpretation and topographic compensation of conifer canopy self-shadowing, Remote sensing of Environment. 112(10): 3820-3832

12. Kattenborn. T.. M. Snerlich. K. Bataua and B. Koch. 2014. Automatic single tree detection in plantations using UAV-based photogrammetric point clouds. The International Archives of Photogrammetry, Remote Sensing and Spatial Information Sciences, 40(3): 139.

13. Koch. B.. U. Hevder and H. Weinacker. 2006. Detection of individual tree crowns in airborne lidar data. Photogrammetric Engineering \& Remote Sensing. 72(4): 357-363.

14. Laliberte. A.S.. A. Rango. J.E. Herrick. E.L. Fredrickson and L. Burkett. 2007. An obiect-based image analvsis approach for determining fractional cover of senescent and green vegetation with digital plot photography. Journal of Arid Environments, 69(1): 1-14.

15. Lin, Y., M. Jiang, Y. Yao, L. Zhang and J. Lin. 2015. Use of UAV oblique imaging for the detection of individual trees in residential environments. Urban Forestry and Urban Greening, 14: 404-412.

16. McNeil, B., J. Pisek, H. Lepisk and E. Flamenco. 2016. Measuring leaf angle distribution in broadleaf canopies using UAVs. Agricultural and Forest Meteorology, 218: 204-208.

17. Mlambo, R., I.H. Woodhouse, F. Gerard and K. Anderson. 2017. Structure from Motion (SfM) photogrammetry with drone data: A low cost method for monitoring greenhouse gas emissions from forests in developing countries. Forests, 8(3): 1-20. 
18. Mohan. M. C.A. Silva. C. Klauherg. P. Jat. G. Catts. A. Cardil. A.T. Hudak and M. Dia. 2017. Individual Tree Detection from Unmanned Aerial Vehicle (UAV) Derived Canopy Height Model in an Open Canopy Mixed Conifer forest. Forest, 8(9): 1-17.

19. Moselou, M. 2012. Assessment of kNN inventory method in estimating the biometric features of wild pistachio trees (Case study: Wild Pistachio Research Forest, Fars). M.Sc. Thesis, Shiraz University. $155 \mathrm{pp}$ (In Persian).

20. Næsset, E. 2002. Predicting forest stand characteristics with airborne scanning laser using a practical two-stage procedure and field data. Remote Sensing Environment, 80: 88-99.

21. Puliti, S., T. Gobakken, H.O. Ørka and E. Næsset. 2017. Assessing 3D point clouds from aerial photographs for species-specific forest inventories. Scandinavian journal of forest research, 32(1): 6879.

22. Rafieyan, O., A.A. Darvishsefat, S. Babaii and A. Mattaji. 2011. Identification of tree species using object-based classification of Digital Aerial Images in the Northern forests of Iran (Case study: Chamestan-Nur). Iranian Journal of Remote Sensing and GIS, 4(2): 63-74 (In Persian).

23. Rafieyan, O., A.A. Darvishsefat, S. Babaii Kafaki and A. Mataji. 2010. Evaluation of pixel-based and object-based classification of aerial images to identify tree species (Case Study: silviculture Chamestan Noor). Journal of Forestry, 1(3): 35-47 (In Persian).

24. Rafieyan, O., A.A. Darvishsefat and M. Namiranin. 2005. Determination of variations in the range of northern forests of the country between 73-80 years by using images etm (case study in Babil forests). Journal of Water and Soil Science Journal of Science and Technology of Agriculture and Natural Resources. 3(10) (In Persian).

25. Saarinen. N.. M. Vastaranta. R. Näsi. T. Rosnell. T. Hakala. E. Honkavaara. M.A. Wulder. V. Luoma. A.M. Tommaselli. N.N. Imai and E.A. Ribeiro. 2018. Assessing Biodiversitv in Boreal Forests with UAV-Based Photogrammetric Point Clouds and Hyperspectral Imaging. Remote Sensing, 10(2): 338.

26. Schiewe, J. 2002. Segmentation of high-resolution remotely sensed data concepts, application and problems. Proceeding of Symposium on Geospatial Theory, Processing and Applications, Ottawa, Canada, 34(4): 235-242.

27. Shabani Pour, M., A.A. Darvishsefat and O. Rafeyan. 2014. Study The Possibility of Identifying Tree Species In Digital Aerial Images Based Object Classification. Journal of the Forest and Wood Products, 67(1): 21-32 (In Persian).

28. Shataee, Sh. 2003. Investigation of the possibility of forest types Mapping using satellite data (Case study: Kheyroud-kenar forest in north of iran), Ph.D. Thesis, University of Tehran Press, Tehran. pp. 44-53 (In Persian).

29. Snerlich. M.. T. Kattenhorn. B. Koch and G. Kattenborn. 2014. Potential of unmanned aerial vehicle based photogrammetric point clouds for automatic single tree detection. Available onlilne: http://www. dgpf. de/neu/Proc2014/proceedings/papers/Beitrag270. pdf (accessed on 15 January 2015).

30. Suomalainen, J., N. Anders, S. Iqbal, G. Roerink, J. Franke, P. Wenting, D. Hünniger, H. Bartholomeus, R. Becker and L. Kooistra. 2014. A lightweight hyperspectral mapping system and photogrammetric processing chain for unmanned aerial vehicles. Remote Sensing, 6(11): 1101311030 .

31. Tang, L. and G. Shao. 2015. Drone remote sensing for forestry research and practices. J. For. Res. 26: 791-797.

32. Torres-Sanchez, F., F. Lopez-Granados and J.M. Pena. 2015. An automatic object-based method for optimal thresholding in UAV image: Application for vegetation detection in herbaceous crops. Computers and Electronics in Agriculture, 114: 43-52.

33. Torresan, C., A. Berton, F. Carotenuto, S.F. Di Gennaro, B. Gioli, A. Matese, F. Miglietta, C. Vagnoli, A. Zaldei and L. Wallace. 2016. Forestry applications of UAVs in Europe: A review. International Journal of Remote Sensing, 38(8-10): 1-21.

34. Vega, F., F. Ramírez, M. Siaz and F. Rosua. 2015. Multi-temporal imaging using an unmanned aerial vehicle for monitoring a sunflower crop. Biosystems Engineering Journal. 132: 19-27.

35. Wallace. L.. A. Lucieer and C.S. Watson. 2014. Evaluating tree detection and segmentation routines on verv high resolution UAV LiDAR data. IEEE Transactions on Geoscience and Remote Sensing, 52(12): 7619-7628.

36. Wang, Y., J. Hyyppa, X. Liang, H. Kaartinen, X. Yu, E. Lindberg, J. Holmgren, Y. Qin, C. Mallet and A. Ferraz. 2016. International Benchmarking of the Individual Tree Detection Methods for Modeling 3-D Canopy Structure for Silviculture and Forest Ecology Using Airborne Laser Scanning. IEEE Trans. Geosci. Remote Sens, 54: 5011-5027.

37. Wang, L., P. Gong and G.S. Biging. 2004. Individual tree-crown delineation and treetop detection in high-spatial-resolution aerial imagery. Photogramm. Eng. Remote Sens, 70: 351-357.

38. Xiang, H. and L. Tian. 2010. Method for automatic georeferencing Aerial remote sensing (RS) images from an unmanned aerial vehicle (UAV) platform. Biosystems Engineering j. 108: 104-113.

39. Yu, Q., P. Gong, N. Clinton, G. Biging, M. Kelly and D. Schirokauer. 2006. Object-based detailed vegetation classification with airborn high spatial resolution remote sensing imagery. Photogrammetric Engineering and Remote Sensing, 72(7): 799-811.

40. Yu, X., J. Hyyppä, M. Karjalainen, K. Nurminen, K. Karila, M. Vastaranta, V. Kankare, H. Kaartinen, M. Holopainen and E. Honkavaara. 2015. Comparison of laser and stereo optical, SAR and InSAR point clouds from air-and space-borne sources in the retrieval of forest inventory attributes. Remote Sensing, 7: 15933-15954.

41. Zarco-Tejada, P.J., R. Diaz-Varela, V. Angileri and P. Loudjani. 2014. Tree height quantification using very high resolution imagery acquired from an unmanned aerial vehicle (UAV) and automatic 3D photo-reconstruction methods. European Journal of Agronomy, 55: 89-99. 


\title{
Detection of Tree Species in Mixed Broad-Leaved Stands of Caspian Forests Using UAV Images (Case study: Darabkola Forest)
}

Milad Pourahmad ${ }^{1}$, Jafar Oladi ${ }^{2}$ and Asghar Fallah ${ }^{3}$

1- Ph.D. Student of Forestry, Faculty of Natural Resources, Sari Agricultural Sciences and Natural Resources University, (Corresponding author: miladpourahmad@yahoo.com)

2 and 3- Associate Professor, Department of Forestry, Faculty of Natural Resources, Sari Agricultural Sciences and Natural Resources University

Received: May 8, 2018

Accepted: June 11, 2018

\begin{abstract}
Unmanned aerial vehicles (UAVs) images have high spatial resolution. They are a valuable source of information for mapping land cover and thematic information, particularly in the identification of tree species. The aim of this study was to investigate the capability of drone images and the base object method for detecting tree species in the Hyrcanian forests. For this purpose, part of an area in parcel 24 of district one in Mazandaran Darabkola forest was selected. The ground truth map was prepared through accurate recording with geographic coordinate's algorithm using distance and azimuth in MATLAB software. Proper processing was done on the images and classification performed on images at three flight height; 55, 75 and 100 meters in two categories of one-step and hierarchical classifications. In object-based classification, the nearest neighbor method was used to classify three species. The accuracy of the maps derived from classifications was evaluated using 50\% of the ground truth map. The results showed that the map of the hierarchical classification by the object based method at a flight height of 55 meters has the best ability to detect tree species in the three heights. These comparisons showed Kappa's coefficient of 0.81 accuracy of tree species classification in 55meter height by UAV.
\end{abstract}

Keywords: Forest classification, Object-based, Tree detection, UAVs 